\title{
Rare Genetic Variants Associated With Myocardial Fibrosis: Multi-Ethnic Study of Atherosclerosis
}

OPEN ACCESS

Edited by:

Jose Vargas,

MedStar Georgetown University Hospital, United States

Reviewed by:

Jan Haas,

Heidelberg University

Hospital, Germany

Roddy Walsh

Academic Medical

Center, Netherlands

Syamal Bhattacharya,

University of Tennessee Health

Science Center (UTHSC),

United States

*Correspondence: Joao A. C. Lima jlima@jhmi.edu

Specialty section:

This article was submitted to Cardiovascular Genetics and Systems

Medicine,

a section of the journal

Frontiers in Cardiovascular Medicine

Received: 29 October 2021

Accepted: 18 January 2022

Published: 21 February 2022

Citation:

Shabani M, Dutta D,

Ambale-Venkatesh B, Post WS,

Taylor KD, Rich SS, Wu CO, Pereira NL, Shah SJ, Chatterjee N, Rotter Jl, Arking DE and Lima JAC

(2022) Rare Genetic Variants Associated With Myocardial Fibrosis: Multi-Ethnic Study of Atherosclerosis.

Front. Cardiovasc. Med. 9:804788.

doi: 10.3389/fcvm.2022.804788

\begin{abstract}
Mahsima Shabani ${ }^{1}$, Diptavo Dutta ${ }^{2}$, Bharath Ambale-Venkatesh ${ }^{3}$, Wendy S. Post ${ }^{1}$, Kent D. Taylor ${ }^{4}$, Stephen S. Rich ${ }^{5}$, Colin O. Wu ${ }^{6}$, Naveen L. Pereira ${ }^{7}$, Sanjiv J. Shah ${ }^{8}$, Nilanjan Chatterjee ${ }^{2}$, Jerome I. Rotter ${ }^{4}$, Dan E. Arking ${ }^{9}$ and Joao A. C. Lima ${ }^{1,3 *}$

${ }^{1}$ Division of Cardiology, Department of Medicine, School of Medicine, Johns Hopkins University, Baltimore, MD, United States, ${ }^{2}$ Department of Biostatistics, Johns Hopkins Bloomberg School of Public Health, Baltimore, MD, United States, ${ }^{3}$ Department of Radiology, Johns Hopkins University, Baltimore, MD, United States, ${ }^{4}$ Department of Pediatrics, The Institute for Translational Genomics and Population Sciences, The Lundquist Institute for Biomedical Innovation at Harbor-University of California, Los Angeles (UCLA) Medical Center, Torrance, CA, United States, ${ }^{5}$ Center for Public Health Genomics, University of Virginia, Charlottesville, VA, United States, ${ }^{6}$ Office of Biostatistics Research, National Heart, Lung, and Blood Institute, National Institutes of Health, Bethesda, MD, United States, ${ }^{7}$ Department of Cardiovascular Medicine, Mayo Clinic, Rochester, MN, United States, ${ }^{8}$ Division of Cardiology, Feinberg Cardiovascular Research Institute, Northwestern University Feinberg School of Medicine, Chicago, IL, United States, ${ }^{9}$ Department of Genetic Medicine, McKusick-Nathans Institute, Johns Hopkins University School of Medicine, Baltimore, MD, United States
\end{abstract}

Background: Rare pathogenic variants in cardiomyopathy (CM) genes can predispose to cardiac remodeling or fibrosis. We studied the carrier status for such variants in adults without clinical cardiovascular disease (CVD) in whom cardiac MRI (CMR)derived measures of myocardial fibrosis were obtained in the Multi-Ethnic Study of Atherosclerosis (MESA).

Objectives: To identify $\mathrm{CM}$-associated pathogenic variants and assess their relative prevalence in participants with extensive myocardial fibrosis by CMR.

Methods: MESA whole-genome sequencing data was evaluated to capture variants in CM-associated genes $(n=82)$. Coding variants with a frequency of $<0.1 \%$ in gnomAD and 1,000 Genomes Project databases and damaging/deleterious effects based on in-silico scoring tools were assessed by ClinVar database and ACMG curation guidelines for evidence of pathogenicity. Cases were participants with high myocardial fibrosis defined as highest quartile of extracellular volume (ECV) or native T1 time in T1-mapping $\mathrm{CMR}$ and controls were the remainder of participants.

Results: A total of 1,135 MESA participants had available genetic data and phenotypic measures and were free of clinical CVD at the time of CMR. We identified 6,349 rare variants in CM-associated genes in the overall MESA population, of which six pathogenic/likely pathogenic (P/LP) variants were present in the phenotyped subpopulation. The genes harboring $\mathrm{P} / \mathrm{LP}$ variants in the case group were $M Y H 7$, CRYAB, and SCN5A. The prevalence of P/LP rare variants in cases was higher than controls (5 in 420 [1.1\%] vs. 1 in 715 [0.1\%], $p=0.03$ ). We identified two MYBPC3 Variants of Unknown Significance (VUS)s with borderline pathogenicity in the case group. The left ventricle (LV) volume, mass, ejection fraction (EF), and longitudinal and circumferential strain in participants with the variants were not different compared to the overall cohort. 
Conclusions: We observed a higher prevalence of rare potentially pathogenic CM associated genetic variants in participants with significant myocardial fibrosis quantified in CMR as compared to controls without significant fibrosis. No cardiac structural or functional differences were found between participants with or without P/LP variants.

Keywords: cardiomyopathy, genetics, fibrosis, magnetic resonance imaging, T1, interstitial, rare

\section{INTRODUCTION}

Myocardial fibrosis is the accumulation of extracellular matrix in the myocardial tissue due to the negligible regenerative capacity of the myocardium (1). It contributes to the pathology of several heart diseases, including dilated and hypertrophic cardiomyopathies (CM) (1). The profibrotic state is an initial step in the process of CM development followed by fibrosis visible in cardiac MRI and evident morphologic changes related to CM (2).

Although, the current gold-standard for detection and quantification of myocardial fibrosis is endomyocardial biopsy, non-invasive imaging techniques such as cardiac MRI (CMR) can provide several indirect markers of myocardial fibrosis (3, 4). Harmonic Phase quantification of tagged CMR evaluates ventricular strain that can identify ventricular dysfunction due to fibrosis-mediated myocardial stiffening (5). Late-Gadolinium Enhancement (LGE) is widely used to illustrate myocardial scars and the replacement fibrosis in the myocardium, reflected by the accumulation of contrast in myocardial tissue (4). T1 mapping is a more recent technique with higher sensitivity thresholds for myocardial fibrosis identification, using direct measurement of extracellular volume (ECV) fraction of myocardial tissue (4). This method has high specificity in detection of myocardial fibrosis and can be easily measured even in those with normal cardiac function (6). Reports show that longer native T1 values depict higher extent of myocardial fibrosis with a $98 \%$ diagnostic accuracy in patients with cardiomyopathy (7). ECV, calculated by hematocrit and pre- and post-contrast T1 values in blood and myocardium, is an index of remodeling in interstitial and extracellular spaces and is a better indicator of collagen volume fraction than post-contrast T1 value (8).

The known genetic background of cardiomyopathies includes variants/mutations in genes related to cell contractility, sarcomere proteins, calcium homeostasis, cytoskeleton, and metabolic pathways, which predispose to changes in ventricular structure, function, and remodeling (9-11). Myocardial fibrosis occurs in CM as well as other myocardial pathologies, contributing to structural and functional changes. Previous genome-wide association studies (GWAS) identified tens of variants associated with CMR-based cardiac structure and function, many of which were located in proximity to Mendelian CM genes including TTN, CDKN1A, BAG3, SH2B3, MYH6, and $M Y H 7$ (12, 13). However, whether rare pathogenic/likely pathogenic $(\mathrm{P} / \mathrm{LP})$ variants in $\mathrm{CM}$ genes are associated with myocardial fibrosis, a predecessor of evident morphologic changes in CM, is unknown. Early identification of pathogenic variants in CM-related genes not only would call for a stricter surveillance in CM prevention and progression in proband and relatives (14), but also identifies the target population for the novel yet promising treatment methods $(15,16)$.

In this study, we aimed to determine if rare P/LP variants (minor allele frequency $<0.1 \%$ ) in $\mathrm{CM}$ genes are enriched in participants with high myocardial fibrosis levels. For this analysis, using whole genome sequencing (WGS) data, a custom list of variants in a cardiomyopathy-related gene panel were called using a bioinformatics pipeline designed based on frequency, location of variant, and its predicted deleterious effect. Cases with a high level of myocardial fibrosis were selected and the distribution of rare pathogenic variants were compared between cases and the remainder of cohort. Finally, other CMRrelated phenotypic measures were evaluated in the carriers of these variants.

\section{METHODS}

\section{Study Population and Case Definition}

The Multi-Ethnic Study of Atherosclerosis (MESA) was initiated in 2000 with a baseline sample of over 6,814 individuals aged 45-84 years, out of whom 4,632 had whole genome sequencing through participation in the TOPMed consortium. MESA includes 38\% White, 28\% African American, 23\% Hispanic, and 11\% Chinese American participants recruited from six US field centers (17). CMR late gadolinium enhancement and T1 mapping studies were performed during the 5th MESA exam (2010-11). A total of 1,345 people underwent contrast-enhanced CMR and T1 mapping. The tagging MRI protocol was applied to 3,100 participants with cardiac MRI in exam 5 and global circumferential strain (GCS) was calculated. Since this analysis is an individual-based analysis, no missing data were imputed and participants lacking outcome or covariate data were excluded.

\section{Myocardial Fibrosis Measurement and Case Selection}

The MRI protocol for the assessment of myocardial fibrosis was previously described (18). Native T1 and ECV derived from T1-mapping MRI were used as direct surrogates of interstitial myocardial fibrosis in this study. T1 mapping studies include estimation of $\mathrm{T} 1$ times at pre-contrast (native) phase and 12and 25-min post gadolinium contrast injection. ECV fraction and partition coefficient were calculated. A single breath-hold ECG-synchronized Modified Look-Locker Inversion recovery (MOLLI) approach was used to assess T1 times. The LGE method identified hyperenhancement areas evident in images recorded $15 \mathrm{~min}$ after a bolus of gadolinium injection. Hyperenhanced areas are reported as the presence of focal scars (binomial variable). 


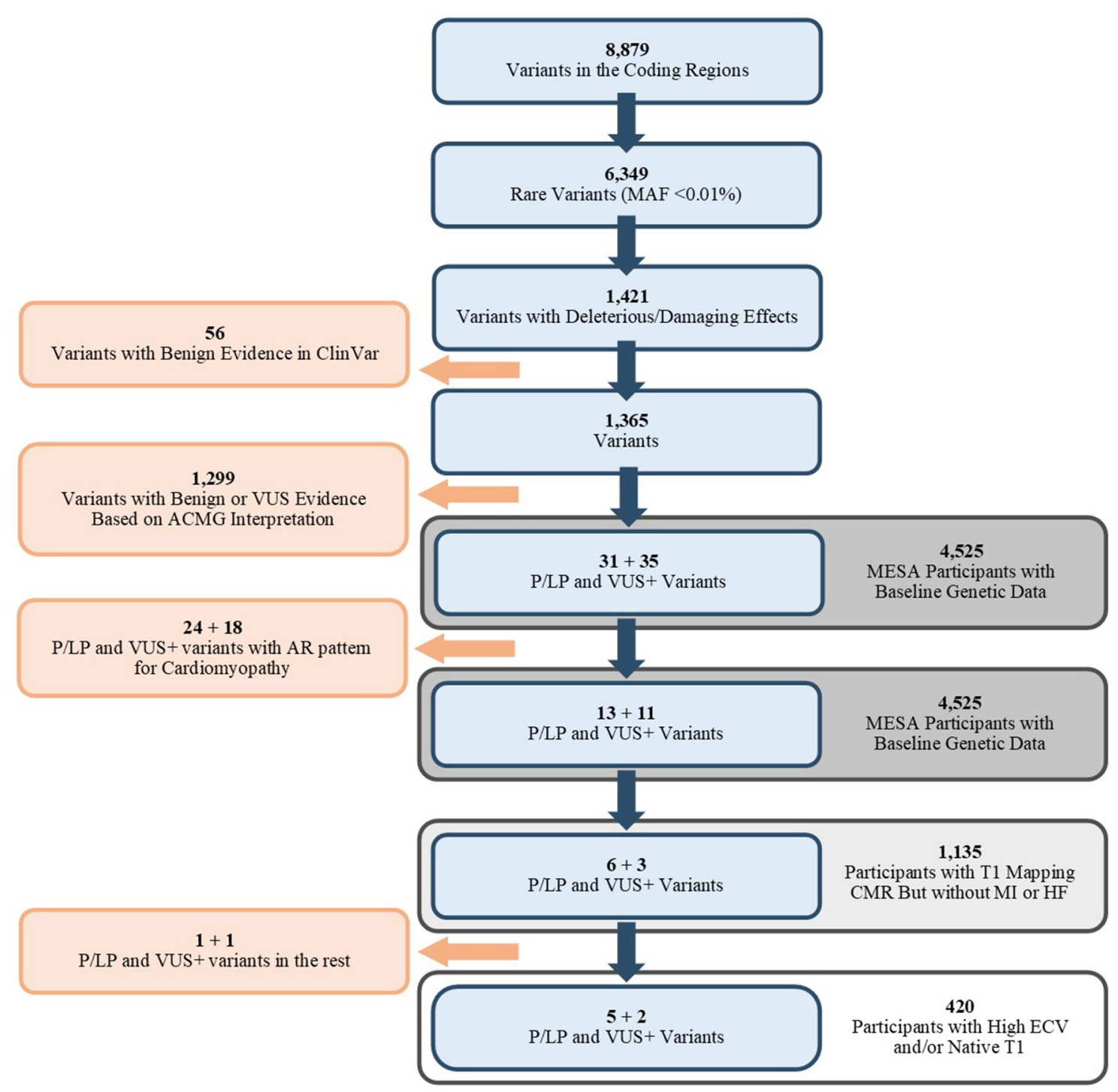

FIGURE 1 | Flowchart of selected variants (Blue) and the associated population. MAF, Mean Allele Frequency; ACMG, American College of Medical Genetics and Genomics; P/LP, Pathogenic/Likely Pathogenic; VUS, Variant of Unknown Significance; VUS+, Variants that were VUS without adjustment of ACMG/AMP criteria; AR, Autosomal Recessive; CMR, Cardiac MRI; MI, Myocardial Infarction; HF, Heart Failure; ECV, Extracellular Volume.

Participants with a history of myocardial infarction (MI) or heart failure (HF) were excluded to filter out participants with a potential replacement fibrosis. Cases were individuals with high myocardial fibrosis defined as the highest quartile of ECV in T1 mapping or highest quartile of native $\mathrm{T} 1$ in the total population with available CMR measurements. Further information on the CMR or echocardiography techniques and analyses is available in the Supplementary Material. The speckle-tracking echocardiography was performed at MESA exam 6 by the Northwestern University Echocardiography Core Lab (NUECL, Chicago, Illinois) (19). The average of the myocardial strain measured in apical 4-, 3-, and 2-chamber views was reported as the LV global longitudinal strain (GLS).

\section{Gene Sequencing}

Whole genome sequencing was performed in DNA samples obtained at the first MESA exam (2000-2002) as part of the Trans-Omic for Precision Medicine (TOPMed) program of National Heart, Lung, and Blood Institute (NHLBI) (20). There were 4,632 participants who underwent sequencing, of whom $107(2.3 \%)$ were excluded due to withdrawal of consent for genomic analyses, enrollment despite preexisting cardiovascular disease, excess DNA contamination, mean sequencing coverage $<30 \times$, or sample duplicates-resulting a final dataset of 4,525 individuals with sequencing data available for the current analysis. Variants were called using Genome Analysis Toolkit HaplotypeCaller software. The sensitivity of the selected variant 
quality score recalibration threshold was $99.8 \%$ for singlenucleotide polymorphisms as empirically assessed using HapMap controls with known genotypes included in the sequencing call set. In total, 1,164 participants had both the variant calls and the T1 mapping phenotypic measures described above.

\section{Bioinformatics Pipeline}

The variant calls were initially filtered to capture any variant in $82 \mathrm{CM}$-related genes (Supplementary Table 1). Only nonmonomorphic variants in the phenotyped subsample were retained for subsequent analysis. These variants were annotated for a spectrum of genomic, regulatory, and other features using the Variant Effect Predictor (VEP version 86) pipeline developed by Ensembl (21). The worst consequence of the variant was chosen across all transcripts (Transcript database: Ensembl/GENCODE v26). The selection pipeline was designed to further narrow down the list of annotated variants based on following criteria: (1) Located in coding and canonical splice site donor and acceptor sites; (2) Frequency of $<0.1 \%$ based on gnomAD (v2.1.1) and 1,000 genomes; (3) Non-synonymous; (4) Deleterious (-or no prediction score) predictive effect using PolyPhen and CADD, or damaging effect (-or no prediction score) using SIFT and FATHMM scoring tools; (5) Non-benign evidence in the ClinVar database (Supplementary Table 1). Resultant variants were assessed by the ACMG/Association of Molecular Pathology (AMP) benign/pathogenic variant classification criteria blinded to the case/control status or any other phenotype information, and those with P/LP interpretation were selected (22). The interpretation platforms of InterVar and Varsome were used to decrease reader-based biases (23, 24). VUSs (Variant of Uncertain Significance) with borderline pathogenicity (likely pathogenic after adjustment of ACMG interpretation based on potentially supporting phenotype) were also reported (VUS+).

\section{Statistical Analysis}

The number of rare variants was compared between the cases and the remainder of the population. The mean values of ECV, GLS, GCS, and left ventricle (LV) anatomical measures [including LV mass index, LV end-diastolic volume (EDV) index, LV endsystolic volume (ESV) index, LV mass to volume ratio (MVR), and ejection fraction (EF)], and prevalence of positive LGE was compared between cases harboring the variants vs. cases who did not and the controls. The related quartile of above-mentioned ventricular features was reported for cases with a P/LP variant and their estimated measures in cases were illustrated relative to the distribution in the entire cohort.

Significance for hypotheses testing was set at $<0.05$ level. All analyses were completed using $\mathrm{R}$ statistical programming software, version 3.6.2. Based on our initial calculation, a sample size of 420 cases would provide a power of $>80 \%$ to detect a $1.5 \%$ difference in prevalence of variants in the cases vs. controls with a significance level of $P<0.05$.
TABLE 1 | Variable distribution among cases vs. controls.

\begin{tabular}{lllr}
\hline Variable & Cases $(\boldsymbol{n}=\mathbf{4 2 0})$ & Controls $(\boldsymbol{n}=\mathbf{7 1 5})$ & $\boldsymbol{P}$-value \\
\hline Age at MRI & $68.6(9.1)$ & $67.0(8.4)$ & $\mathbf{0 . 0 0 2}$ \\
Sex & & & \\
Female & $265(63.1 \%)$ & $277(38.7 \%)$ & $\mathbf{0 . 0 0 1}$ \\
Male & $155(36.9 \%)$ & $438(61.3 \%)$ & \\
Race & & & \\
White & $232(55.2 \%)$ & $379(53.0 \%)$ & \\
African American & $105(25.0 \%)$ & $151(21.1 \%)$ & \\
Chinese American & $43(10.2 \%)$ & $71(9.9 \%)$ & 0.12 \\
Hispanic & $40(9.5 \%)$ & $114(15.9 \%)$ & 0.58 \\
BMl (kg/m²) & $28.1(5.7)$ & $28.6(4.8)$ & $<\mathbf{0 . 0 0 1}$ \\
SBP (mmHg) at CMR & $121.8(19.6)$ & $121.2(18.4)$ & 0.80 \\
DBP (mmHg) at CMR & $67.0(10.0)$ & $69.3(9.3)$ & $<\mathbf{0 . 0 0 1}$ \\
Diabetes mellitus & $65(15.7 \%)$ & $113(15.9 \%)$ & $<\mathbf{0 . 0 0 1}$ \\
ECV & $29.4(2.5)$ & $25.6(1.8)$ & \\
Native T1 & $1,014(37.1)$ & $957.2(30.7)$ & \\
\hline BM & & & \\
& & &
\end{tabular}

BMI, Body mass index; SBP, Systolic blood pressure; DBP, Diastolic blood pressure; ECV, Extracellular volume. Values marked in bold show significant associations.

\section{RESULTS}

A total of 1,164 MESA participants had available genetic data and the T1 mapping measurements available. Participants who had a history of MI or HF before the time of CMR acquisition ( $n$ $=29$ ) were excluded from the analysis (Figure 1). There was a total of 420 participants (37\%) who had either an ECV level in the fourth quartile only $(\mathrm{ECV}>28.8, n=139)$, a native T1 time in the fourth quartile only (native T1 $>1,006 \mathrm{~ms}, n=139$ ), or both $(n=142)$. Median age at the time of CMR exam was 69 years in cases with $63 \%$ females and 67 years in controls (39\% female). There were 55 and $53 \%$ of the cases and controls who were white (Table 1).

Nine P/LP variants or VUS+ were identified among the 1,135 participants (Figure 1). The details of ACMG/AMP interpretation of variants are available in Supplementary Table 2. After unblinding of the case status, 7 variants (5 P/LP, 2 VUS+) in 4 genes (MYH7, CRYAB, MYBPC3, and SCN5A) were observed in 7 cases, and 2 variants ( $1 \mathrm{P} / \mathrm{LP}, 1$ VUS+) in 2 distinct genes (MYL2, TNNT2) were observed in the remaining 715 participants (Table 2). Among the 7 participants in the case group, two had non-cardiovascular death and one developed HF during follow-up.

The prevalence of any P/LP variant or VUS+ in the CM genes was higher in the case group $(1.7 \%[7 / 420])$ vs. the controls $(0.3 \%$ [2/715], $p=0.01)$. The prevalence of any P/LP variants was higher in the case group $(1.2 \%[5 / 420])$ vs. the controls $(0.1 \%$ [1/715], $p=0.03)$.

One case with a P/LP variant in MYH7 had evidence of scar in the LGE analysis (Table 3). All of the cases had an EF of higher than $45 \%$ (min: $51.3 \%$ ). The values of indexed LV mass, LV EDV, LV ESV, and LV MVR were heterogeneous in the carriers of CM variants (Figure 2). However, there were no significant difference in LV end-diastolic mass to volume ratio (1.1 vs. 1.0, $p=0.54), \mathrm{LV}$ EF (62.1 vs. $62.0, p=0.97)$, and other anatomical 
TABLE 2 | Cases/Controls with pathogenic/likely pathogenic variants and VUS+ in cardiomyopathy genes with AD pattern of inheritance.

\begin{tabular}{|c|c|c|c|c|c|c|c|c|c|c|c|}
\hline & Age & Sex, race & $\begin{array}{l}\text { Phenotype } \\
\text { criteria }\end{array}$ & Gene & Type & Variant & Transcript & $\begin{array}{l}\text { ClinVar } \\
\text { interpretation }\end{array}$ & $\begin{array}{l}\text { ACMG } \\
\text { interpretation }\end{array}$ & $\begin{array}{l}\text { gnomAD AF in } \\
\text { ethnic group }\end{array}$ & $\begin{array}{l}\text { Associated } \\
\text { disease }\end{array}$ \\
\hline \multicolumn{12}{|c|}{ Cases } \\
\hline C1 & $50-60$ & F, African & High ECV & SCN5A & Missense & $3: 38613773 G>A$ & p.Arg225Trp & $\mathrm{P} / \mathrm{LP}$ & Pathogenic & $1.28 \mathrm{e}-4$ & DCM, LVNC \\
\hline \multirow[t]{2}{*}{ C2 } & $70-80$ & $F$, African & High Native T1 & CRYAB & Missense & $11: 111908822 \mathrm{G}>\mathrm{A}$ & Ap.Arg157His & VUS & $\begin{array}{l}\text { Likely } \\
\text { pathogenic }\end{array}$ & $6.15 e-5$ & DCM, LVNC \\
\hline & & & High ECV & & & & & & & & \\
\hline C3 & $50-60$ & M, Chinese & High Native T1 & CRYAB & Start codon loss & $11: 111911722 \mathrm{G}>\mathrm{A}$ & Ap.Met1lle & Conflicting & $\begin{array}{l}\text { Likely } \\
\text { pathogenic }\end{array}$ & $9.84 \mathrm{e}-4$ & DCM, LVNC \\
\hline \multirow[t]{2}{*}{ C4 } & $80-90$ & F, White & High Native T1 & MYH7 & Missense & $14: 23424839 G>A$ & p.Arg870His & Pathogenic & $\begin{array}{l}\text { Likely } \\
\text { pathogenic }\end{array}$ & $1.55 \mathrm{e}-5$ & $\begin{array}{l}\text { HCM, DCM, } \\
\text { LVNC }\end{array}$ \\
\hline & & & High ECV & & & & & & & & \\
\hline C5 & $60-70$ & $\mathrm{M}$, African & High Native T1 & MYH7 & Missense & $14: 23429037 \mathrm{C}>\mathrm{T}$ & p.Arg442His & Conflicting & $\begin{array}{l}\text { Likely } \\
\text { pathogenic }\end{array}$ & $6.15 e-5$ & $\begin{array}{l}\text { HCM, DCM, } \\
\text { LVNC }\end{array}$ \\
\hline C6 & $50-60$ & M, Hispanic & High ECV & МYBPCЗ & Missense & $11: 47337543 G>A$ & p.Arg817Gln & Conflicting & VUS+ & $2.83 e-5$ & $\begin{array}{l}\text { HCM, DCM, } \\
\text { LVNC }\end{array}$ \\
\hline \multirow[t]{2}{*}{$\mathrm{C} 7$} & $70-80$ & M, White & High Native T1 & МYBPCЗ & Missense & $11: 47337792 \mathrm{G}>\mathrm{A}$ & p.Val771Met & Conflicting & VUS+ & $1.76 e-5$ & $\begin{array}{l}\text { HCM, DCM, } \\
\text { LVNC }\end{array}$ \\
\hline & & & High ECV & & & & & & & & \\
\hline \multicolumn{12}{|c|}{ Controls } \\
\hline $\mathrm{N} 1$ & $50-60$ & M, Hispanic & - & MYL2 & Missense & $12: 110914290 \mathrm{C}>\mathrm{T}$ & Tp.Gly57Glu & VUS & $\begin{array}{l}\text { Likely } \\
\text { pathogenic }\end{array}$ & $2.89 e-5$ & $\mathrm{HCM}$ \\
\hline N2 & $60-70$ & M, White & - & TNNT2 & Missense & $1: 201365261 \mathrm{G}>A$ & p.Ala114Val & Conflicting & VUS+ & $1.76 e-5$ & $\begin{array}{l}\text { HCM, DCM, } \\
\text { RCM, LVNC }\end{array}$ \\
\hline
\end{tabular}

*VUS+: Variants that were VUS without adjustment of ACMG/AMP criteria.

ECV, Extracellular volume; DCM, Dilated cardiomyopathy; LVNC, Left ventricular non-compaction; HCM, Hypertrophic cardiomyopathy; RCM, Restrictive cardiomyopathy. 
TABLE 3 | Individual-based description of global circumferential strain and LV anatomical features in cases with P/LPNUS+ variants.

\begin{tabular}{|c|c|c|c|c|c|c|c|c|c|c|c|}
\hline \#Case & Age category & Phenotype criteria & Gene & Variant & GCS & LV ESV & LV EDV & LV mass & LV ED-MVR & LV EF & LGE scar \\
\hline $\mathrm{C} 2$ & 70-80, F & High Native T1 & CRYAB & $11: 111908822 \mathrm{G}>\mathrm{A}$ & Q1 & Q3 & Q2 & Q1 & Q1 & $>45 \%$ & No \\
\hline C3 & 50-60, M & High Native T1 & CRYAB & $11: 111911722 \mathrm{G}>\mathrm{A}$ & Q2 & Q2 & Q3 & Q3 & Q3 & $>45 \%$ & No \\
\hline \multirow[t]{2}{*}{ C4 } & 80-90, F & High Native T1 & MYH7 & $14: 23424839 G>A$ & Q3 & Q1 & Q1 & Q2 & Q4 & $>45 \%$ & No \\
\hline & & High ECV & & & & & & & & & \\
\hline C6 & 50-60, M & High ECV & МYBPCЗ & $11: 47337543 G>A$ & Q1 & Q4 & Q4 & Q4 & Q2 & $>45 \%$ & No \\
\hline \multirow[t]{2}{*}{$\mathrm{C} 7$} & $70-80, M$ & High Native T1 & МYВРСЗ & $11: 47337792 G>A$ & NA & NA & NA & NA & NA & NA & No \\
\hline & & High ECV & & & & & & & & & \\
\hline
\end{tabular}

GCS, Global circumferential strain; LV, Left ventricle; ESV, End-systolic volume; EDV, End-diastolic volume; ED-MVR, End-diastolic mass-to-volume ratio; EF, Ejection fraction; LGE, Late gadolinium enhancement; ECV, Extracellular volume.

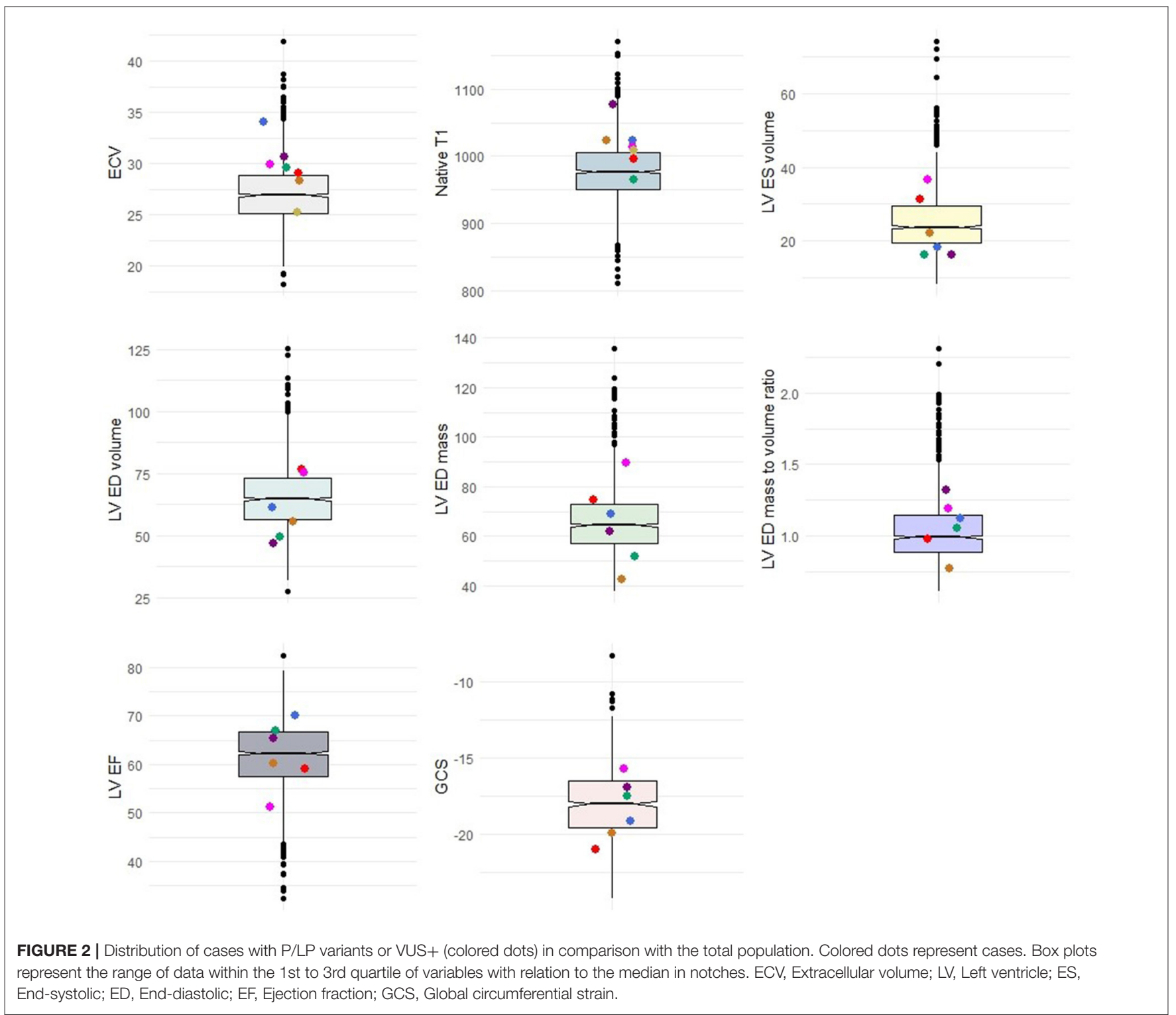


TABLE 4 | Comparison of LV anatomic features between cases with CM variants and without variants, as well as cases with CM variants and the controls.

\begin{tabular}{|c|c|c|c|c|c|}
\hline Variable & Cases with variant $(n=7)$ & Cases without variants $(n=413)$ & $P$-value & Controls $(n=715)$ & $P$-value \\
\hline ECV (\%) & $29.5(2.6)$ & $29.5(2.6)$ & 0.99 & $25.5(1.9)$ & 0.007 \\
\hline Native T1 (ms) & $1,015.1(33.7)$ & 1,013.2 (39.1) & 0.88 & 955.9 (31.0) & 0.003 \\
\hline LV EDM (g/mm²) & $65.0(13.1)$ & $64.3(14.3)$ & 0.91 & $66.4(12.0)$ & 0.84 \\
\hline LV EDV $\left(\mathrm{mL} / \mathrm{mm}^{2}\right)$ & $60.9(12.7)$ & $65.3(13.7)$ & 0.44 & $65.2(13.1)$ & 0.45 \\
\hline LV ESV $\left(\mathrm{mL} / \mathrm{mm}^{2}\right)$ & $23.5(8.6)$ & $25.0(8.3)$ & 0.67 & $25.1(7.5)$ & 0.68 \\
\hline LV ED-MVR (g/mL) & $1.1(0.2)$ & $1.0(0.2)$ & 0.54 & $1.0(0.2)$ & 0.74 \\
\hline LV EF (\%) & $62.1(6.7)$ & $62.0(7.6)$ & 0.97 & $61.8(6.6)$ & 0.92 \\
\hline GCS by CMR (\%) & $18.3(2.0)$ & $18.3(2.4)$ & 0.96 & $17.9(2.2)$ & 0.57 \\
\hline GLS by ST-echo (\%) & $19.0(2.1)$ & $20.1(2.9)$ & 0.61 & $19.7(2.7)$ & 0.74 \\
\hline
\end{tabular}

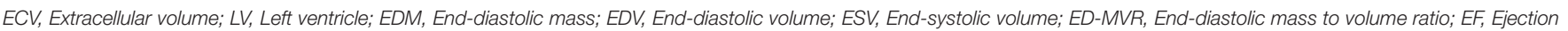

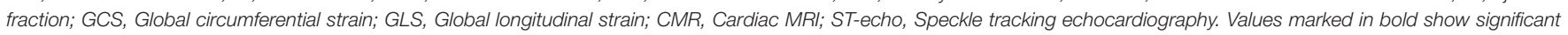
associations.

LV features between cases with CM variants and cases with no CM variants (Table 4). Likewise, there was no difference in anatomical LV features between cases with pathogenic variants and controls (Table 4). Furthermore, there was no difference in functional LV measures, including GCS and GLS, between cases with pathogenic variants and either cases without pathogenic variants (GCS: 18.3 vs. $18.3, p=0.96$; GLS: 19.0 vs. $20.1, p=0.61$ ) or controls (GCS: 18.3 vs. $17.9, p=0.57$, GLS: 19.0 vs. $19.7, p$ $=0.74$ ). Figure 2 illustrates the distribution of cardiac structural and functional variables in cases with $\mathrm{P} / \mathrm{LP}$ or VUS+ variants compared to the distribution of each variable in the entire cohort.

\section{DISCUSSION}

In this study, we observed rare P/LP/VUS+ variants in 4 genes associated with cardiomyopathy (MYH7, CRYAB, SCN5A, and $M Y B P C 3)$ in individuals without a history of MI or HF and with extensive myocardial fibrosis indicated by CMR. The prevalence of $\mathrm{P} / \mathrm{LP}$ variants in this group was higher $(1.1 \%)$ than those with lower myocardial fibrosis $(0.1 \%)$. On average, participants with rare $\mathrm{P} / \mathrm{LP}$ or VUS+ variants did not have a significantly different LV volume, mass, EF, and longitudinal and circumferential strain at the time of CMR, compared to the overall cohort.

Identification of individuals who carry P/LP variants can be used to screen for genetic risk for cardiomyopathy and consequent cardiac dysfunction or HF in the clinically asymptomatic stage $(25,26)$. Although the cost and the processing time of genetic sequencing is decreasing, the rare frequency of these variants in the general population, without reliable clinical predictors, makes genetic testing a less ideal screening method. However, the yield of genetic testing may improve in a more focused target population with a higher prevalence of pathogenic variants, in this case, asymptomatic individuals with high myocardial fibrosis in CMR. Identification of pathogenic variants in these participants can subsequently be used for family screening and risk prediction $(27,28)$.

Previous reports suggest myocardial fibrosis as a marker of risk for future cardiac dysfunction (29, 30). Cardiac autopsy identified myocardial fibrosis as the primary cause of $3.6 \%$ of sudden cardiac death in victims of non-ischemic CM
(31). Further DNA testing revealed that more than $10 \%$ of individuals had a P/LP variant in CM associated genes. None of the individuals harboring the genetic variants had anatomical findings in the heart autopsy suggestive of CM (31). Likewise, although some of the participants with variants in this study had cardiac structural and functional findings suggestive of cardiomyopathy, on average, there was no difference between variant careers and the non-carrier cases or the controls in ventricular volume, mass, EF, and strain values. Therefore, identification of CM variants associated with CMR detected myocardial fibrosis may help stratify asymptomatic patients at risk for sudden death. Whether such patients are more likely to exhibit disease penetrance or develop CM remains to be proven.

Mutations in the genes expressing sarcomere protein components including MYH7, MYBPC3, and MYL2 are found in a significant number of patients with dilated or hypertrophic cardiomyopathy and thus, pathogenic variants in these genes are recommended by ACMG to be reported even as a secondary or incidental finding in genome or exome sequencing (25). We observed P/LP variants in MYBPC3 and MYH7 in four participants with high interstitial fibrosis, yet not all had evident myocardial dysfunction. Previous studies have shown that myocardial fibrosis, measured through the level of serum Cterminal pro-peptide of type I procollagen (PICP), was observed in the carriers of pathogenic sarcomere variants in individuals with or without overt cardiomyopathy (2). A comprehensive CMR analysis of $133 \mathrm{HCM}$ patients showed that patients with sarcomere variants had higher ECV and number of segments with LGE compared to patients with mitochondria-related mutations or no mutations (32). Cardiomyopathy caused by variation in sarcomere genes such as MYBPC3 have been associated with an inflammatory phenotype and subsequent fibrosis (33), and whether the fibrosis detected by CMR in these patients is a result of inflammation remains to be proven.

Finding a well-powered sample size for the identification of rare variants associated with certain diseases with genomewide significance is a struggle. Guided sampling used in this study where the tails of phenotype distribution are selected aides in increasing the power of these studies (34). However, even though the higher prevalence of P/LP variants in participants 
with extensive myocardial fibrosis suggests the carrier status for these variants as a potential risk factor, we should note that this study does not have sufficient power to detect a strong association between the observed variants and myocardial fibrosis. In particular, the study population consisted of participants who not only had no CVD at baseline but also survived at least for 10 years (to the 5th MESA exam), making them a healthier population. Thus, we believe that the real prevalence of rare variants with a pathogenic effect in myocardial fibrosis in people with extensive myocardial fibrosis could be even higher. The cases in our study were older and were femaledominant compared to controls, which could also account for the increased fibrosis observed in the case group (30). However, it is well-known that epigenetic factors in the setting of genetic susceptibility contribute to the manifestation of CM (35).

The deep-coverage WGS data provided in the multiethnic Trans-Omics for Precision Medicine (TOPMed) genomic resource provides a convenient platform for the detection of rare variants not detectable in similar genome-wide databases $(20,36)$. On the other hand, MESA offers solid predictors of subclinical CM through its unique CMR-based assessments of cardiac fibrosis, the T1 mapping. To facilitate the comparison, we grouped participants with any LP/P variant together. Nevertheless, the restricted number of carriers of P/LP variants limited this study from evaluating the course of progression of cardiac dysfunction and event rate in these carriers. Moreover, despite using a multi-ethnic cohort, readers should note that the result of this study does not apply to ethnic groups not involved in the MESA study, including South Asians and the non-Chinese Asian population. A limitation of running the variants found in a group of individuals through bioinformatics pipelines is the inability to interpret a few criteria for pathogenicity or benign status of variant. For example, de novo status (PS2) or segregation in relatives (BS4, PP1) cannot be determined with no family studies.

Additional longitudinal assessment of carriers of these variants and validation studies in other cardiovascular cohorts may set the stage for the use of myocardial fibrosis with CM gene-panel assessment as a preventive and personalized cardiology tool in people at risk for cardiomyopathy. Early preventative care in these carriers coupled with effective control of comorbidities may attenuate the development of HF (14). Moreover, with the discovery of novel and promising therapeutic approaches, including sarcomere protein inhibitors (15) and base editors (16), carriers of pathogenic variants in CM-related genes can be treated, if not cured.

\section{DATA AVAILABILITY STATEMENT}

Publicly available datasets were analyzed in this study. This data can be found here: MESA website (https://www.mesa-nhlbi. $\operatorname{org} /)$.

\section{ETHICS STATEMENT}

The studies involving human participants were reviewed and approved by all field centers and core labs of the MESA study including: Columbia University, New York Johns Hopkins University, Baltimore Northwestern University, Chicago UCLA, Los Angeles University of Minnesota, Twin Cities Wake Forest University, Winston Salem University of Washington Coordinating Center University of Vermont Laboratory UCLA Medical Center Research and Education Institute New England Medical Center The Lundquist Institute for Biomedical Innovation at Harbor-UCLA Medical Center University of Virginia Broad Institute of MIT and Harvard National Heart, Lung, and Blood Institute. The patients/participants provided their written informed consent to participate in this study.

\section{AUTHOR CONTRIBUTIONS}

MS, DD, NC, and DA analyzed the genetic data. MS and DD drafted the manuscript. SS, NP, JR, KT, and SR helped with genetic and phenotype data acquisitions. JR, SR, DA, WP, BA-V, and JL helped with the study design. CW, DA, and JL supervised the biostatistical analysis. JL, DA, CW, WP, JR, and BA-V revised the draft. All authors contributed to the article and approved the submitted version.

\section{FUNDING}

WGS for the TOPMed program was supported by the National Heart, Lung and Blood Institute (NHLBI). WGS for 'NHLBI TOPMed: Multi-Ethnic Study of Atherosclerosis (MESA) (phs001416.v1.p1) was performed at the Broad Institute of MIT and Harvard (3U54HG003067-13S1). Centralized read mapping and genotype calling, along with variant quality metrics and filtering were provided by the TOPMed Informatics Research Center (3R01HL117626-02S1, contract HHSN268201800002I). Phenotype harmonization, data management, sample-identity QC, and general study coordination, were provided by the TOPMed Data Coordinating Center (3R01HL-120393; U01HL-120393; contract HHSN268180001I). The MESA project is conducted and supported by the National Heart, Lung, and Blood Institute (NHLBI) in collaboration with MESA investigators. Support for MESA is provided by contracts 75N92020D00001, HHSN268201500003I, N01-HC-95159, 75N92020D00005, N01-HC-95160, 75N92020D00002, N01-HC-95161, 75N92020D00003, N01-HC-95162, 75N92020D00006, N01-HC95163, 75N92020D00004, N01-HC-95164, 75N92020D00007, N01-HC-95165, N01-HC-95166, N01-HC-95167, N01-HC95168, N01-HC-95169, UL1-TR-000040, UL1-TR-001079, and UL1-TR-001420. Also supported in part by the National Center for Advancing Translational Sciences, CTSI grant UL1TR001881, and the National Institute of Diabetes and Digestive and Kidney Disease Diabetes Research Center 
(DRC) grant DK063491 to the Southern California Diabetes Endocrinology Research Center.

\section{ACKNOWLEDGMENTS}

The authors thank the other investigators, the staff, and the participants of the MESA study and the Trans-Omics in Precision Medicine (TOPMed) program for their valuable contributions.

\section{REFERENCES}

1. Kong P, Christia P, Frangogiannis NG. The pathogenesis of cardiac fibrosis. Cell Mol Life Sci. (2014) 71:549-74. doi: 10.1007/s00018-013-1349-6

2. Ho CY, López B, Coelho-Filho OR, Lakdawala NK, Cirino AL, Jarolim P, et al. Myocardial fibrosis as an early manifestation of hypertrophic cardiomyopathy. N Engl J Med. (2010) 363:55263. doi: 10.1056/NEJMoa1002659

3. Mewton N, Liu CY, Croisille P, Bluemke D, Lima JA. Assessment of myocardial fibrosis with cardiovascular magnetic resonance. J Am Coll Cardiol. (2011) 57:891-903. doi: 10.1016/j.jacc.2010.11.013

4. Ambale-Venkatesh B, Lima JA. Cardiac MRI: a central prognostic tool in myocardial fibrosis. Nat Rev Cardiol. (2015) 12:1829. doi: 10.1038/nrcardio.2014.159

5. Venkatesh BA, Donekal S, Yoneyama K, Wu C, Fernandes VR, Rosen BD, et al. Regional myocardial functional patterns: quantitative tagged magnetic resonance imaging in an adult population free of cardiovascular risk factors: the multi-ethnic study of atherosclerosis (MESA). J Magn Reson Imaging. (2015) 42:153-9. doi: 10.1002/jmri.24749

6. Gyöngyösi M, Winkler J, Ramos I, Do QT, Firat H, Mcdonald K, et al. Myocardial fibrosis: biomedical research from bench to bedside. Eur J Heart Fail. (2017) 19:177-91. doi: 10.1002/ejhf.696

7. Puntmann VO, Voigt T, Chen Z, Mayr M, Karim R, Rhode K, et al. Native T1 Mapping in differentiation of normal myocardium from diffuse disease in hypertrophic and dilated cardiomyopathy. JACC Cardiovasc Imaging. (2013) 6:475-84. doi: 10.1016/j.jcmg.2012.08.019

8. Haaf P, Garg P, Messroghli DR, Broadbent DA, Greenwood JP, Plein S. Cardiac T1 Mapping and Extracellular Volume (ECV) in clinical practice: a comprehensive review. J Cardiovasc Magn Reson. (2016) 18:89. doi: 10.1186/s12968-016-0308-4

9. Jacoby D, McKenna WJ. Genetics of inherited cardiomyopathy. Eur Heart J. (2012) 33:296-304. doi: 10.1093/eurheartj/ehr260

10. Toepfer CN, Garfinkel AC, Venturini G, Wakimoto H, Repetti G, Alamo L, et al. Myosin sequestration regulates sarcomere function, cardiomyocyte energetics, and metabolism, informing the pathogenesis of hypertrophic cardiomyopathy. Circulation. (2020) 141:828-42. doi: 10.1161/CIRCULATIONAHA.119.042339

11. Walsh R, Offerhaus JA, Tadros R, Bezzina CR. Minor hypertrophic cardiomyopathy genes, major insights into the genetics of cardiomyopathies. Nat Rev Cardiol. (2021). doi: 10.1038/s41569-021-00608-2. [Epub ahead of print].

12. Aung N, Vargas JD, Yang C, Cabrera CP, Warren HR, Fung $\mathrm{K}$, et al. Genome-wide analysis of left ventricular image-derived phenotypes identifies fourteen loci associated with cardiac morphogenesis and heart failure development. Circulation. (2019) 140:1318-30. doi: 10.1161/CIRCULATIONAHA.119.041161

13. Pirruccello JP, Bick A, Wang M, Chaffin M, Friedman S, Yao J, et al. Analysis of cardiac magnetic resonance imaging in 36,000 individuals yields genetic insights into dilated cardiomyopathy. Nat Commun. (2020) 11:2254. doi: 10.1038/s41467-020-15823-7

14. Hershberger RE, Givertz MM, Ho CY, Judge DP, Kantor PF, Mcbride KL, et al. Genetic evaluation of cardiomyopathy: a clinical practiceresource of the American College of Medical Genetics and Genomics (ACMG). Genet Med. (2018) 20:899-909. doi: 10.1038/s41436-0180039-Z
We gratefully acknowledge the studies and participants who provided biological samples and data for TOPMed.

\section{SUPPLEMENTARY MATERIAL}

The Supplementary Material for this article can be found online at: https://www.frontiersin.org/articles/10.3389/fcvm. 2022.804788/full\#supplementary-material

15. Olivotto I, Oreziak A, Barriales-Villa R, Abraham TP, Masri A, Garcia-Pavia P, et al. Mavacamten for treatment of symptomatic obstructive hypertrophic cardiomyopathy (EXPLORER-HCM): a randomised, double-blind, placebo-controlled, phase 3 trial. Lancet. (2020) 396:759-69. doi: 10.1016/S0140-6736(20)31792-X

16. Ma S, Jiang W, Liu X, Lu WJ, Qi T, Wei J, et al. Efficient correction of a hypertrophic cardiomyopathy mutation by ABEmax-NG. Circ Res. (2021) 129:895-908. doi: 10.1161/CIRCRESAHA.120.318674

17. Bild DE, Bluemke DA, Burke GL, Detrano R, Diez Roux AV, Folsom AR, et al. Multi-Ethnic study of atherosclerosis: objectives and design. Am J Epidemiol. (2002) 156:871-81. doi: 10.1093/aje/kwf113

18. Liu CY, Liu YC, Wu C, Armstrong A, Volpe GJ, Van Der Geest RJ, et al. Evaluation of age-related interstitial myocardial fibrosis with cardiac magnetic resonance contrast-enhanced T1 mapping: MESA (MultiEthnic Study of Atherosclerosis). J Am Coll Cardiol. (2013) 62:12807. doi: 10.1016/j.jacc.2013.05.078

19. Patel RB, Freed BH, Beussink-Nelson L, Allen NB, Konety SH, Post WS, et al. Associations of cardiac mechanics with exercise capacity. J Am Coll Cardiol. (2021) 78:245-57. doi: 10.1016/j.jacc.2021.04.082

20. Taliun D, Harris DN, Kessler MD, Carlson J, Szpiech ZA, Torres R, et al. Sequencing of 53,831 diverse genomes from the NHLBI TOPMed Program. Nature. (2021) 590:290-9. doi: 10.1038/s41586-021-03205-y

21. McLaren W, Gil L, Hunt SE, Riat HS, Ritchie GRS, Thormann A, et al. The ensembl variant effect predictor. Genome Biol. (2016) 17:122. doi: 10.1186/s13059-016-0974-4

22. Richards S, Aziz N, Bale S, Bick D, Das S, Gastier-Foster J, et al. Standards and guidelines for the interpretation of sequence variants: a joint consensus recommendation of the American College of Medical Genetics and Genomics and the Association for Molecular Pathology. Genet Med. (2015) 17:405424. doi: 10.1038/gim.2015.30

23. Li Q, Wang K. InterVar: clinical interpretation of genetic variants by the 2015 ACMG-AMP Guidelines. Am J Hum Genet. (2017) 100:26780. doi: 10.1016/j.ajhg.2017.01.004

24. Kopanos C, Tsiolkas V, Kouris A, Chapple CE, Albarca Aguilera M, Meyer $\mathrm{R}$, et al. VarSome: the human genomic variant search engine. Bioinformatics. (2019) 35:1978-80. doi: 10.1093/bioinformatics/bty897

25. Musunuru K, Hershberger RE, Day SM, Klinedinst NJ, Landstrom AP, Parikh VN, et al. Genetic testing for inherited cardiovascular diseases: a scientific statement from the American Heart Association. Circulation. (2020) 13:e000067. doi: 10.1161/HCG.0000000000000067

26. Rosenbaum AN, Agre KE, Pereira NL. Genetics of dilated cardiomyopathy: practical implications for heart failure management. Nat Rev Cardiol. (2020) 17:286-297. doi: 10.1038/s41569-019-0284-0

27. Ho CY, Day SM, Ashley EA, Michels M, Pereira AC, Jacoby D, et al. Genotype and lifetime burden of disease in hypertrophic cardiomyopathy: insights from the Sarcomeric Human Cardiomyopathy Registry (SHaRe). Circulation. (2018) 138:1387-98. doi: 10.1161/CIRCULATIONAHA.117.033200

28. Verdonschot JAJ, Hazebroek MR, Krapels IPC, Henkens MTHM, Raafs A, Wang $\mathrm{P}$, et al. Implications of genetic testing in dilated cardiomyopathy. Circulation. (2020) 13:476-87. doi: 10.1161/CIRCGEN.120.003031

29. Liu T, Song D, Dong J, Zhu P, Liu J, Liu W, et al. Current understanding of the pathophysiology of myocardial fibrosis and its quantitative assessment in heart failure. Front Physiol. (2017) 8:238. doi: 10.3389/fphys.2017.00238

30. Ambale-Venkatesh B, Liu C-Y, Liu Y-C, Donekal S, Ohyama Y, Sharma RK, et al. Association of myocardial fibrosis and cardiovascular events: 
the multi-ethnic study of atherosclerosis. Eur Heart J. (2018) 20:168176. doi: 10.1093/ehjci/jey140

31. Junttila MJ, Holmström L, Pylkäs K, Mantere T, Kaikkonen K, Porvari $\mathrm{K}$, et al. Primary myocardial fibrosis as an alternative phenotype pathway of inherited cardiac structural disorders. Circulation. (2018) 137:2716726. doi: 10.1161/CIRCULATIONAHA.117.032175

32. Chung H, Kim Y, Park C-H, Kim J-Y, Min PK, Yoon YW, Kim TH, et al. Effect of sarcomere and mitochondria-related mutations on myocardial fibrosis in patients with hypertrophic cardiomyopathy. J Cardiovasc Magn Reson. (2021) 23:18. doi: 10.1186/s12968-021-00718-3

33. Lynch TLT, Ismahil MA, Jegga AG, Zilliox MJ, Troidl C, Prabhu $\mathrm{SD}$, et al. Cardiac inflammation in genetic dilated cardiomyopathy caused by MYBPC3 mutation. J Mol Cell Cardiol. (2017) 102:8393. doi: 10.1016/j.yjmcc.2016.12.002

34. Barnett IJ, Lee S, Lin X. Detecting rare variant effects using extreme phenotype sampling in sequencing association studies. Genet Epidemiol. (2013) 37:14251. doi: 10.1002/gepi.21699

35. Giudicessi JR, Shrivastava S, Ackerman MJ, Pereira NL. Clinical impact of secondary risk factors in TTN-mediated dilated cardiomyopathy. Circulation. (2021) 14:e003240. doi: 10.1161/CIRCGEN.120. 003240

36. Kowalski MH, Qian H, Hou Z, Rosen JD, Tapia AL, Shan Y, et al. Use of $>100,000$ NHLBI Trans-Omics for Precision Medicine (TOPMed) Consortium whole genome sequences improves imputation quality and detection of rare variant associations in admixed
African and Hispanic/Latino populations. PLoS Genet. (2019) 15:e1008500. doi: 10.1371/journal.pgen.1008500

Conflict of Interest: The authors declare that the research was conducted in the absence of any commercial or financial relationships that could be construed as a potential conflict of interest.

The handling editor declared a past co-authorship with one of the authors with the authors WP, KT, SR, JR, and JL.

Publisher's Note: All claims expressed in this article are solely those of the authors and do not necessarily represent those of their affiliated organizations, or those of the publisher, the editors and the reviewers. Any product that may be evaluated in this article, or claim that may be made by its manufacturer, is not guaranteed or endorsed by the publisher.

Copyright (C) 2022 Shabani, Dutta, Ambale-Venkatesh, Post, Taylor, Rich, Wu, Pereira, Shah, Chatterjee, Rotter, Arking and Lima. This is an open-access article distributed under the terms of the Creative Commons Attribution License (CC BY). The use, distribution or reproduction in other forums is permitted, provided the original author(s) and the copyright owner(s) are credited and that the original publication in this journal is cited, in accordance with accepted academic practice. No use, distribution or reproduction is permitted which does not comply with these terms. 